\title{
Developing a national registry of veterans with spinal cord dysfunction: experiences and implications
}

\author{
Greg Samsa ${ }^{1,2,3,6}$, Helen Hoenig ${ }^{4,5,6}$, John Carswell ${ }^{9}$, Richard Sloane ${ }^{2}$, Catherine Ross Bovender ${ }^{2}$, \\ Carol VanDeusen Lukas, ${ }^{7,8}$ and Ron D Horner ${ }^{2,6}$ \\ ${ }^{1}$ Center for Health Policy Research and Education, ${ }^{2}$ Department of Medicine, ${ }^{3}$ Department of Community and Family \\ Medicine, ${ }^{4}$ Center for Aging, Duke University, Durham NC, USA; ${ }^{5}$ Physical Medicine and Rehabilitation Service, \\ ${ }^{6}$ Center for Health Services Research in Primary Care, Department of Veterans Affairs Medical Center, Durham NC, \\ USA; ${ }^{7}$ Management Decision Research Center, Department of Veterans Affairs, Boston MA, USA; ${ }^{8}$ Health Services \\ Department, School of Public Health, Boston University, Boston MA, USA; ${ }^{9}$ Paralyzed Veterans of America, \\ Washington DC, USA
}

\begin{abstract}
We describe the development of a registry of veterans with spinal cord dysfunction who have been treated within the Department of Veterans Affairs health care facilities. The registry departs from the function and structure of traditional registries by a more extensive utilization of advances in computer technology; in particular, by its reliance upon computerized record linkage and by its association with a set of computer-based clinical management and reporting tools. We discuss some of the applications of the registry to research for persons with spinal cord dysfunction as well as implications that our experiences provide for developing other registries of persons with disabilities.
\end{abstract}

Keywords: spinal cord injury; spinal cord dysfunction; registry; data base

\section{Introduction}

A registry is 'a file of documents containing uniform information about individual persons, collected in a systematic and comprehensive way, in order to serve a predetermined purpose. ${ }^{1}$ Disease registries-for example, the registry operated by the United States Model Spinal Cord Injury (SCI) Systems program, ${ }^{2}-$ have greatly contributed to our knowledge regarding the prevalence, natural history, cost, and sequelae of diseases. With continued advances in computer technology, the capability for obtaining and managing information for registries has vastly increased, and the potential applications for registries have multiplied.

We recently developed and implemented an ongoing national registry of American veterans with spinal cord dysfunction (SCD) who receive care at Department of Veterans Affairs (VA) facilities. The VA registry departs from the design of traditional registries by a more extensive utilization of advances in computer technology; in particular, by its reliance upon computerized record linkage and by its association with a set of computer-based clinical management and reporting tools. We first describe our experiences in developing a SCD registry within

Correspondence: G Samsa the VA. Then, we discuss some of the distinctive features and applications of computer-based registries.

\section{Background}

\section{Terminology}

Spinal cord dysfunction can be caused by either traumatic events (eg, injury), or by non-traumatic events (eg, diseases such as multiple sclerosis). SCD denotes spinal cord dysfunction regardless of cause. SCI denotes SCD of traumatic cause. SCD of nontraumatic cause is referred to as such.

Organization of care for veterans with SCD

In the VA, care for persons with traumatic SCI is organized through the Spinal Cord Injury Service, which has designated 22 VA Medical Centers (VAMCs) as specialized SCI Centers. SCI Centers handle approximately two-thirds of inpatient visits for persons with SCI. ${ }^{3}$ Some SCI Centers also care for veterans with SCD regardless of cause, while others care for persons with SCI only.

At the approximately 150 VAMCs without a specialized SCI Center, care is typically provided by an SCI Primary Care Team which includes a physician, a nurse, and an SCI Coordinator. The 
SCI Coordinator, who is usually a social worker, is responsible for most contact and follow-up.

VAMCs have similar hospital data management systems, all of which are based upon the VA's Decentralized Hospital Computer Program (DHCP). All hospital computer systems maintain a standard set of data elements, which are supplemented by local additions as desired.

\section{Registry development process}

Historically, planning for veterans with SCD has been hampered by inadequate information. ICD-9 diagnostic codes used to identify persons with SCD were often applied inconsistently, as was a special data field that purportedly identified persons with SCI. ${ }^{4}$ Administrative files did not record basic information about SCD (eg, date of onset or injury, injury level and completeness), functional status variables (eg, activities of daily living) or information about met and unmet needs. A standardized information resource was needed.

Registry development began with an agreement about the registry's basic design and inclusion criteria. The registry is intended to include all veterans who (a) have SCD of either traumatic or non-traumatic cause, and (b) utilize VA health care services. All VAMCs have local registries that follow SCD veterans treated at those facilities. Local registries can upload information into a national database linked to other VA national databases. Registry development was predicted upon the premise that having computer-based tools for clinical management linked to local computer systems would give providers an incentive to populate local registries, which in turn would help ensure the comprehensiveness of the national database.

An expert panel, which includes SCI physicians, SCI nurses, SCI coordinators, VA administrators, software developers, and patient advocates, was convened and began by identifying the operational goals of the registry. One goal was to provide clinicians with a means to more easily track persons with SCD and to more easily organize clinical information on individual patients. A second goal was to provide administrators, policy makers, and advocates with population-level data to aid in procuring and organizing services for SCD veterans. A final goal was to provide researchers with an easily accessible list of patients from which to draw nationally representative samples.

In order to proceed expeditiously, programmers modified software created for another VA registry. The initial version of SCD registry software had modules for case registration and reporting. The case registration module supported the input of identifying information (eg, social security number) and basic SCD-specific clinical data (eg, date of injury or onset of disease). The reporting module linked the case registration module with the much more extensive data maintained on local hospital computer systems.
Modifying existing software facilitated creation of a generic registry within constraints of time and budget. However, the initial version of registry software was not widely used. Barriers to use included inadequate communication with users, overly technical training manuals, some data elements that were either difficult to collect or not in a format desired by clinicians, and reporting formats which were not sufficiently specific to patients with SCD. The expert panel recommended modifications to the software intended to increase ease of use, to increase clinical relevance, and to obtain information which the SCI Service could use as performance measures. The case registration and reporting modules were modified, and functional status and clinical modules were added. Training materials were redesigned.

Developers also noted that users such as SCI Coordinators had little time for case finding. Accordingly, we 'seeded' local registries through an independent case-finding operation. Registry developers used information from VA administrative files (eg, ICD-9 codes, visits to SCI bed sections) to identify approximately 50000 veterans thought likely to have SCD and mailed surveys to each. Approximately 25000 surveys were returned, of which approximately 18000 reported SCD. Surveys were keyed at a central location and the results were then electronically transferred to local registries. Case finding is ongoing through local initatives.

\section{Membership criteria}

The registry's inclusion criteria (Appendix 1) are based upon diseases or conditions whose pathophysiology either primarily or secondarily affects the spinal cord, spinal canal, or cauda equina. Classification of traumatic SCI is straightforward. For non-traumatic SCD, diagnoses are divided into two groups. The first group, which includes conditions such as multiple sclerosis, contains all persons with that diagnosis regardless of level of impairment. (This decision was made because impairment level tends to change over time - for example, the impairment attributable to multiple sclerosis could at any time be high, minimal or even absent). In the second group of diagnoses, which includes conditions such as malignant neoplasms that have metastasized to the spine and spinal cord, impairment is required as a condition for registry membership.

\section{Data elements}

The registry's case registration module is divided into two components - basic registration and basic health care data. The basic registration component includes elements such as name, social security number, date and cause of SCD, injury level and completeness, and multiple sclerosis subtype (Appendix 2). The basic health care data component includes information about providers, site of initial rehabilitation, and annual 
rehabilitation visits. Some data elements offer the option of simplified reporting. For example, injury level may be reported as tetraplegia/paraplegia, and completeness may be reported as complete/incomplete.

The functional status module (Appendix 2) allows the local user to select among various measures. While most measures are applied in the usual fashion, we have modified the Functional Independence Measure $(\mathrm{FIM})^{5}$ in order to be based upon either observer- or patient-report. That is, the FIM may be performed in the traditional manner by a clinician or clinicians, using the full FIM scale with 18 items (13 motor items and five cognitive items), each having seven levels. Alternatively, functional status may be based upon patient self-report, which includes only the 13 motor items of the FIM and utilizes four rather than seven response categories. Initial validation studies of the above self-reported measure have been encouraging. ${ }^{6}$

\section{Reports}

The above data elements can be uploaded to a national database linked with other national VA databases. In addition to the above, local users can make use of almost every data element maintained on their local DHCP system. For example, the registry's reporting capability can be used to identify patients who have not visited the SCI clinic as expected, who have received urgent care, who have been admitted to the hospital with pressure sores, who have out-of-range laboratory values, who have received prosthetic devices, who have future clinic visits scheduled, and so forth. Reports can pertain to individual patients (eg, a schedule of clinic appointments combined with selected laboratory results) or groups of patients (eg, total inpatient hospital days at a facility).

\section{Comment}

We have described our experiences in developing a national VA registry of veterans with SCD. Although by no means trivial, our registry development task was simplified by a number of considerations. First, the VA has a strong organizational commitment to improving the care of persons with SCD. Second, despite local modifications, each VAMC has essentially the same computer system, thus simplifying software design and record linkage. Third, the VA's computerized administrative files already maintain much of the data required for a registry focused on utilization and planning, thus reducing the need for prospective data collection. Finally, the registry is linked with a clinical reporting tool, thus increasing receptivity by clinical users. $^{7}$ Despite these favorable circumstances, and despite the systematic approach to registry development described above, the initial version of the registry did not fully meet its users needs and required significant redesign. The Model Systems Registry underwent a similar redesign. ${ }^{2}$ One implication for future registry developers is the importance of including all users - especially data collectors - in the design process as early as possible. Another implication is the need for timetables and budgets to include the possibility of modification and redesign.

Appendix 3 contains a checklist of questions we considered during the registry development process. Most are self-explanatory and can be applied to registry development in general.

To briefly comment on some of the items in Appendix 3, registries may have various goals including identification of individuals, surveillance, general epidemiology, evaluating services, evaluating treatments, research and patient and provider education. ${ }^{8}$ While all of these were important to the VA, its registry's primary goals were identifying individuals, facilitating planning, and increasing the amount of information easily accessible to the clinician.

Accurate case definition and comprehensive case finding are crucial to the validity of a registry. At its most basic, a registry is a list of cases. However, not every list of cases is a registry. For example, a list of patients having the diagnosis of multiple sclerosis on an administrative file is not a registry because diagnoses on administrative files tend to be inaccurate and incomplete. This list of cases would become a registry with the adoption of a formal protocol for identifying and verifying cases.

The number of data elements which can be collected reliably is limited. Appendix 4 describes the criteria we used to determine whether or not to collect a data element. Whenever possible, registry data are collected as part of ongoing clinical or administrative processes. For example, FIM measurements can be made by clinicians as part of their usual assessment procedures, or might instead be based upon a self-report which can be filled out by the patient while he or she is waiting to see the clinician. Our ability to link local registries with other VA databases was a crucial factor allowing us to limit the number of data elements requiring primary data collection. Thus, although the registry itself contains only a sparse set of variables, it is effectively equivalent to a much larger database because of the large number and variety of data elements already maintained in the local DHCPs and the VA's national administrative files.

\section{Applications}

One noteworthy property of computer-based registries lies in their ability to support multiple types of research. The VA SCD Registry supports research in a number of ways. First, it facilitates recruitment of patients into randomized trials (eg, a randomized trial comparing treatments for multiple sclerosis) by providing a nationally representative list of patients from which to sample. Second, it supports outcomes research by automating follow-up of patients' health service utilization, mortality, and functional status. Third, it supports basic epidemiological research into the natural history of SCD by providing the ability to 
follow changes in functional status over time. (This can be done by forming cohorts to be prospectively followed through the registry, and/or by using the registry to create a representative group of cases to be included in case-control designs). Fourth, the registry plays a crucial role in supporting quality improvement research. For example, the VA's SCI Service uses the registry to measure quality improvement indicators such as the percentage of SCD veterans who have a primary physician, the percentage of SCD veterans who are offered an annual rehabilitation evaluation, the percentage of SCD veterans who have had a functional assessment, and so forth. Not only is the registry used to identify potential quality problems, but it can also track the success of interventions intended to address these problems. Finally, the Registry's ability to link patients with resource utilization helps support research on the cost of care for veterans with SCD. Without a population-based denominator available from a registry, estimates of the cost of care can be biased - for example, because patients using little or no services tend to be overlooked.

A future application of computer-based registries is to accelerate the process of developing comprehensive national and international data bases pertaining to persons with SCD. This was considered in a recent conference, ${ }^{9}$ one of the conclusions of which is that the most feasible way to develop comprehensive international registries involves developing separate computer-based registries that can subsequently be linked. We have demonstrated the ability to successfully link registry data across approximately 170 hospitals, albeit within a single health care system. As computer technology continues to improve, we anticipate that computerized record linkage could be expanded to include multiple health care systems and eventually multiple nations. Furthermore, we anticipate that registry designs based upon record linkage will become increasingly cost-effective in comparison with more traditional registry designs.

The continuing advance of computer technology will change the focus, change the design, and broaden the applicability of registries pertaining to persons with disabilities. One of the challenges of the $21 \mathrm{st}$ century will be translating the additional information available from registries first into improved clinical practice and ultimately into improved outcomes.

\section{Acknowledgements}

We gratefully acknowledge Ms. Annette Jurgelski for editorial assistance. Registry development efforts were jointly funded by the Department of Veterans Affairs and Paralyzed Veterans of America (PVA), a congressionally chartered veterans service organization dedicated to advocacy on behalf of veterans with SCD. The VA's Health Services Research and Development Service, through its Management Decision and Research Center and Durham Field Program, was responsible for registry development.

We acknowledge grant support from the Health Services Research and Development Service, Department of Veterans Affairs and the Paralyzed Veterans of America. Drs. Hoenig and Horner were partially funded by the National Institute of Health, National Institute of Aging, Claude D Pepper Older Americans Center grant 5PG0AG11268.

\section{References}

1 Brooke EM. The Current and Future Use of Registers in Health Information Systems. World Health Organization: Geneva 1974.

2 Stover SL, DeLisa JA, Whiteneck GG (eds). Spinal Cord Injury: Clinical Outcomes from the Model Systems. Aspen Publications: Gaithersburg, MD 1995.

3 Samsa GP, Landsman PB, Hamilton BB. Inpatient hospital utilization among veterans with traumatic spinal cord injury. Archives of Physical Medicine and Rehabilitation 1996; 77: 1037 1043.

4 Samsa GP et al. The development and preliminary testing of an approach to identifying veterans with spinal cord dysfunction (SCD) for a new national registry. Department of Veterans Affairs Health Services Research and Development National Meeting: Washington, DC. April, 1994.

5 Hamilton BB et al. A uniform national data system for medical rehabilitation. In: Fuhrer $\mathrm{M}$ (ed). Rehabilitation Outcomes: Analysis and Measurement. Brookes: Baltimore MD 1987; pp. $137-147$.

6 Hoenig $\mathrm{H}$ et al. A simple, self-reported measure of function for persons with disability. Presented at Gerontological Society of America Annual Scientific Meeting. Washington DC. November, 1996.

7 Levi R, Hultling C, Westgren N. A computer assisted follow up system for spinal cord injury patients. Paraplegia 1994; 32: $736-$ 742 .

8 Goldberg J, Gelfand HM, Levy PS. Registry evaluation methods: A review and case study. Epidemiologic Reviews 1980; 21: $210-$ 220

9 Proceedings of the National SCI Data Conference (National Spinal Cord Injury Association, National Association of Health Data Organizations, Centers for Disease Control, Department of Veterans Affairs, National Institute of Disability and Rehabilitation Research, National Center for Medical Rehabilitation Research and Paralyzed Veterans of America, sponsors). Paralyzed Veterans of America: Washington, DC. 1995

\section{Appendix 1 Membership Criteria}

045

094.0

138

192.2

192.3

198.4

225.3

225.4

324.1

334.0

334.1

334.8

334.9

335.2

336

340

334.0

344.1

344.2

344.6 acute poliomyelitis

Tabes dorsalis

late effects of poliomyelitis

malignant neoplasm of spinal cord malignant neoplasm of spinal meninges secondary malignant neoplasm of meninges secondary benign neoplasm of spinal cord secondary benign neoplasm of spinal meninges intraspinal abscess Friedreich's ataxia hereditary spastic paraplegia other spinocerebellar diseases spinocerebellar disease, unspecified motor neuron disease other diseases of spinal cord multiple sclerosis quadriplegia paraplegia diplegia of upper limbs cauda equina syndrome 
thoracic spondylosis with myelopathy lumbar spondylosis with myelopathy unspecified spondylosis with myelopathy intervertebral disc disorder with myelopathy fracture of vertebral column with SCI

\section{late effects of SCI}

SCI without evidence of spinal bone injury

\section{2}

were the ICD-9 codes used to screen administrative files. Other diagnoses may be included by the provider as warrranted. Some of the above diagnoses (eg, quadriplegia) require evidence of disability due to SCI.

These criteria were developed by a neurologist and reviewed by the registry's expert panel.

\section{Appendix 2 Data Elements}

Registration module: Basic registration

Name

Social security number

Vital status

$\mathrm{SCI} / \mathrm{SCD}$ indicator

SCI network

Registration status

Date of registration

Date of last update

Cause(s) of SCD

Date of SCD onset

Highest level of injury

Completeness of injury

Multiple sclerosis subtype

Registration module: Basic health care data

Site(s) of medical care - SCI Center, VA medical center where care is primarily received, other VA providers, other

non-NA providers

Primary care provider

SCI coordinator

Referral source

Site of initial rehabilitation

Date of discharge from initial rehabilitation

Annual rehabilitation evaluation offered - date

Annual rehabilitation evaluation received - date

Next annual rehabilitation evaluation scheduled - date

Bowel care reimbursement program membership

Bowel care reimbursement program certified

Bowel care reimbursement program provider

Functional module

Activities of daily living-basic

Activities of daily living-instrumental

Need for personal assistance (yes/no, per $24 \mathrm{~h}$, per 2 weeks)

Method of ambulation - walking

Method of ambulation - wheelchair

Functional Independence Measure (FIM) - self-report

Functional Independence Measure (FIM) - observer-based

Functional Assessment Measure (FAM)

Craig Assessment and Reporting Technique (CHART)

Duke University Severity of Illness Index (DUSOI)

Diener Quality of Life Index

Kurtzke Functional Systems Scale (FS)

Kurtzke Expanded Disability Status Scale (EDSS)

Clinical module

ASIA impairment score

ASIA motor score
ASIA sensory score

ASIA neurological levels

ASIA partial preservation - sensory

ASIA partial preservation - motor

Specific body parts affected

Extent of movement

Extent of feeling

Amputation

Head injury

Data from other DHCP components available for reports

Patient demographics (eg, address, telephone, date of birth, age, gender, race, insurance)

Inpatient (eg, date of admission, date of discharge, patient location, admission diagnoses, discharge diagnoses, discharge location)

Outpatient (eg, clinic stops)

Pharmacy

Laboratory

Radiology

Appendix 3 Registry Development Checklist

* Have all stakeholders been identified?

* Have all stakeholders participated in defining registry goals?

* Have all stakeholders agreed to registry goals?

* Have registry goals been clearly defined?

* Have registry goals been clearly communicated to data collectors?

* Has the content of registry reports been defined?

* Have end users been given the opportunity to comment on the format and content of registry reports?

* Do all stakeholders receive reports and/or other products from the registry?

* Do the registry's data elements use standard definitions?

* Have explicit membership criteria been set?

* Does an explicit process exist to assist data collectors in determining the membership criteria of uncertain cases?

* Have all potential sources of cases been identified?

* Will case finding be an ongoing process or a one-time event?

* Has the target population been explicitly identified?

* Have the personnel responsible for case finding been identified?

* Do case finders and other data collectors have an incentive to collaborate?

* Do the personnel responsible for case finding have adequate resources to do so?

* If selected within an existing organization, do case finders have approval to perform case finding?

* Does the registry collect extraneous data elements?

* Are data elements collected as efficiently as possible? (eg, as a by-product of existing clinical or administrative processes)

* Have personnel responsible for quality assurance and oversight been identified?

* Have general functional specifications been received by stakeholders for consistency with registry goals?

* Have technical specifications been reviewed by programmers to ensure consistency?

* Have technical specifications been reviewed by end users to ensure completeness and appropriateness?

* Have the goals and content of training for data collectors been defined?

* Have end users had the opportunity to comment on the form and content of training materials? 
* Is information about data quality fed back to data collectors?

* Does a written data collection protocol exist?

* Has the data collection protocol been pilot tested?

* Do data collectors receive a copy of the data collection protocol?

* Does a written data management protocol exist?

* Has the data management protocol been pilot tested?

* Do data management personnel receive a copy of the data management protocol?

* Do development timetables and budgets provide adequate leeway for testing and redesign if necessary?

* Does the registry have a stable organizational home?

* Does the registry have sufficient development funds?

* Have functions such as data collection, data management, data analysis, quality assurance, coordination, training, and clinical consultation been adequately funded?

* Does the registry have sufficient funds for long-term maintenance?

* Has the registry been adequately publicized?

Note: General specifications describe users and uses; for example, 'the registry will assist clinicians by increasing the amount of patient-specific information which is easily accessible'. Technical specifications involve individual data elements, including possible values, protocols for collection, and responsible individuals. For example, "date of onset will be collected by the SCI Coordinator at the time of registration and is defined as the date of injury for persons with traumatic injury and the date of diagnosis for persons with non-traumatic SCD. If day and month are unknown, these should be reported as missing.'

Appendix 4 Criteria for Determining the Importance of a Data Element

Does the data element satisfy all of the following?

* The data element must be associated with an explicitly stated clinical issue, hypothesis, or research question.

* The data element should be well enough defined to allow a formal data collection protocol to be written.

* A means should exist to accurately collect the data element.

* Data collectors should have an incentive for accurate data collection.

* The data element should not be recorded on another computer-accessible file.

* If clinical, the data element should be sufficiently important to require collection on all patients. 